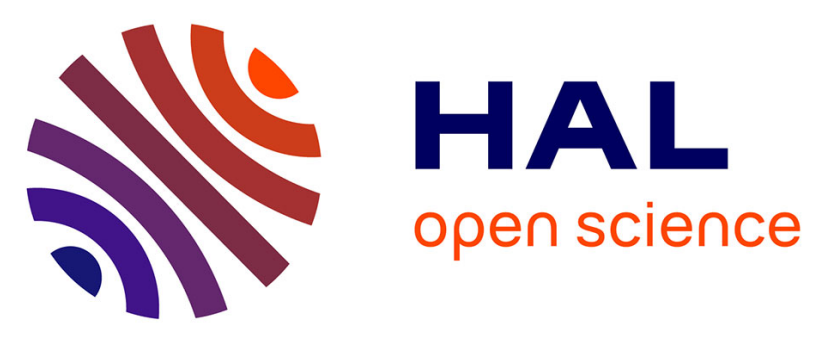

\title{
Direct writing of lateral fluorographene nanopatterns with tunable bandgaps and its application in new generation of moiré superlattice
}

$\mathrm{Hu}$ Li, Tianbo Duan, Soumyajyoti Haldar, Biplab Sanyal, Olle Eriksson, Hassan Jafri, Samar Hajjar-Garreau, Laurent Simon, Klaus Leifer

\section{To cite this version:}

Hu Li, Tianbo Duan, Soumyajyoti Haldar, Biplab Sanyal, Olle Eriksson, et al.. Direct writing of lateral fluorographene nanopatterns with tunable bandgaps and its application in new generation of moiré superlattice. Applied Physics Reviews, 2020, 7 (1), pp.011403. 10.1063/1.5129948 . hal-02881678

\section{HAL Id: hal-02881678 \\ https://hal.science/hal-02881678}

Submitted on 10 Dec 2020

HAL is a multi-disciplinary open access archive for the deposit and dissemination of scientific research documents, whether they are published or not. The documents may come from teaching and research institutions in France or abroad, or from public or private research centers.
L'archive ouverte pluridisciplinaire HAL, est destinée au dépôt et à la diffusion de documents scientifiques de niveau recherche, publiés ou non, émanant des établissements d'enseignement et de recherche français ou étrangers, des laboratoires publics ou privés. 
This article may be downloaded for personal use only. Any other use requires prior permission of the author and AIP Publishing. This article appeared in Li et al., Appl. Phys. Rev. 7, 011403 (2020) (DOI: 10.1063/1.5129948) and may be found at https://aip.scitation.org/doi/ 10.1063/1.5129948

\section{Direct Writing of Lateral Fluorographene Nanopatterns with Tunable}

\section{Bandgaps and Its Application in New Generation of Moiré Superlattice}

$\mathrm{Hu} \mathrm{Li}^{1, \mathrm{a})}$, Tianbo Duan ${ }^{1, \mathrm{a})}$, Soumyajyoti Haldar ${ }^{2,3}$, Biplab Sanyal $^{2}$, Olle Eriksson ${ }^{2,4}, \mathrm{~S}$. Hassan M. Jafri ${ }^{1}$, Samar Hajjar-Garreau ${ }^{5}$, Laurent Simon $^{5}$, and Klaus Leifer ${ }^{1, b)}$

\section{AFFILIATIONS:}

${ }^{1}$ Department of Engineering Sciences, Applied Materials Science, Ångström Laboratory, Uppsala University, 75121 Uppsala, Sweden.

${ }^{2}$ Department of Physics and Astronomy, Materials Theory, Ångström Laboratory, Uppsala University, 75121 Uppsala, Sweden.

${ }^{3}$ Institute of Theoretical Physics and Astrophysics, University of Kiel, D-24098 Kiel, Germany.

${ }^{4}$ School of Science and Technology, Orebro University, 70182 Orebro, Sweden.

${ }^{5}$ Institut de Sciences des Matériaux de Mulhouse, CNRS-UMR 7361, Université de Haute Alsace, 68093 Mulhouse, France.

a) Authors contributed equally to this work. b) Author to whom correspondence should be addressed: Klaus.Leifer@angstrom.uu.se

ABSTRACT: A desirable picture of graphene as an atomic thin "canvas" is to freely draw semiconducting/insulating lateral nanopatterns directly on the semi-metallic graphene surface, which is one of the most looked-for goals for monolayer device fabrications. Here, we have demonstrated a reversible electron beam activated technique, which allows to directly write semiconducting/insulating fluorographene lateral nanopatterns with tunable bandgaps on the graphene surface and a resolution down to 9-15 $\mathrm{nm}$. This approach overcomes the conventional limit of semiconducting $\mathrm{C}_{4} \mathrm{~F}$ in the single-sided fluorination of supported graphene and achieves insulating $\mathrm{C}_{2} \mathrm{~F}$. Moreover, by applying this technique on bilayer graphene, we have shown a new type of rectangular moiré patterns arising from the generated $\mathrm{C}_{2} \mathrm{~F}$ boat/graphene superlattice for the first time. This novel technique constitutes a new approach to fabricate 
graphene-based flexible and transparent electronic nanodevices with the $\mathrm{C}_{\mathrm{x}} \mathrm{F}$ channels utilized as semiconducting or insulating counterpart, and also opens a route towards the tailoring and engineering of electronic properties of such materials in addition to the dominating triangular moiré patterns from graphene/hBN system.

\section{I . INTRODUCTION}

The inherent two-dimensional feature of graphene raises a concept as an atomically thin "canvas", where different functional groups could be deposited onto this canvas similar to an "ink" on the surface leading to distinct materials properties in different areas on the graphene surface $^{1-5}$. As a promising next-generation material in electronics, an ideal picture of graphene is to freely draw the semiconducting/insulating patterns directly on conductive graphene surfaces with nanometer resolution. Fluorine shows superiorities as the "ink" towards this goal, owing to the efficiency in tuning the bandgap and towards achieving a chemically stable product $^{6-10}$. A large variety of techniques has been reported to fluorinate graphene ranging from pure $F_{2}$ exposure to fluorine plasma etching ${ }^{11,12}$. In many works, the synthesis of monolayer fluorographene and stoichiometric fluorographene (perfluorographane, CF) hitherto is carried out by exposing graphene to $\mathrm{XeF}_{2}$ gas $^{7,8,13}$. As for the more applicable supported graphene (e.g. graphene on $\mathrm{SiO}_{2}$ ) where a physical support substrate avoids the mechanical collapsing, the product with the highest fluorine content is semiconducting $\mathrm{C}_{4} \mathrm{~F}$ for the above mentioned plasma based and chemical fluorination pathways ${ }^{7,14}$, and it is still difficult to synthesize insulating fluorographene, e.g. $\mathrm{C}_{2} \mathrm{~F}$ and $\mathrm{CF}$, on a substrate directly. Theoretical studies have predicted that, by tuning the concentration of fluorine ${ }^{15,16}$, the electronic properties of the fluorographene such as the presence of a bandgap can be tailored to meet different requirements. Experimentally, optical band gap measurements have proved the possibility of bandgap opening in the fluorographene ${ }^{13}$, yet the electronic band gap, important for most applications of fluorographene, has rarely been shown ${ }^{17,18}$. 
In this work, we explored graphene fluorination processes, the physical property and structure changes in a rather broad parameter space, both theoretically and experimentally. For the purpose of a well-defined and local synthesis of fluorographene, we have developed a reversible and local electron beam activated fluorination technique, where the nano-sized electron beam induced deposition process cracks the $\mathrm{XeF}_{2}$ precursor gas and atomic fluorine is bound to the graphene surface covalently. This reversible technique is proved here to be very efficient. When it is applied to free-standing graphene, perfluorographane (CF) can be obtained after short synthesis time. Our X-ray photoelectron spectroscopy (XPS) studies of these fluorinated graphene surfaces suggest that for single-sided fluorination on the supported graphene, insulating $\mathrm{C}_{2} \mathrm{~F}$ with a bandgap of $\sim 5.8 \mathrm{eV}$ is obtained which implies that our technique has overcome the conventional limit of semiconducting $\mathrm{C}_{4} \mathrm{~F}$ in the fluorination of supported graphene and achieved the insulating fluorographene. The valence band spectra analysis of the fluorographene of different F-concentration shows that fluorine induces a discernible band opening in the graphene electronic structure and the bandgap of the fluorographene increases with the fluorine coverage. From the theoretical analysis of the fluorographene, we analyze at which ordering conditions a bandgap opening could appear. The ordering of graphene could also be analyzed from the fluorination of bilayer graphene using the same technique. As an impactful observation, a new type of rectangular moiré pattern is for the first time observed between the upmost fluorinated graphene and the bottom unfluorinated graphene layer. This rectangular shaped moiré patterns can be well explained from the atomically locked bilayer $\mathrm{C}_{2} \mathrm{~F}$ boat/graphene superlattices. In turn, these structure analysis results are compared to the theoretical electronic structure analysis of fluorographene.

\section{I . RESULTS AND DISCUSSION}

The schematic picture of the electron beam activated fluorination (EBAF) is shown in Fig. 1A. The core idea of this method is the local creation of $F$ radicals by the electron beam, resulting in the nano-synthesis of fluorographene directly on monolayer graphene under the nanometer 
sized electron beam. For the fluorographene synthesis, graphene was placed under the focused electron beam irradiation ${ }^{19-21}$ and simultaneously, a flux of $\mathrm{XeF}_{2}$ molecules was injected through a gas injection system. Since the electrons in the initial beam is well above the critical energy to dissociate $\mathrm{XeF}_{2}^{22,23}$, the decomposition of the $\mathrm{XeF}_{2}$ molecules was induced, leading to the formation of local $\mathrm{F}$ radicals and finally resulting in the graphene fluorination. Here, two types of graphene samples were used: free-standing graphene $(\mathrm{FG})$ on transmission electron microscopy (TEM) grid and supported graphene (SG) on $\mathrm{SiO}_{2}$ substrate (Fig. 1B and 1C). It is found that the typical saturation time for the fluorination of FG and SG were 10 and 5 minutes for a selected $100 \mu \mathrm{m} \times 100 \mu \mathrm{m}$ area in measured characteristics, receptively.

The XPS spectra of pristine graphene, fluorinated SG and FG samples are compared in Fig. 2A. In the fluorinated FG, the area under the XPS C 1s peak consists to $\sim 90 \%$ of the $\mathrm{C}(-\mathrm{F})$ peak, and $\mathrm{C}\left(\mathrm{sp}^{2}\right.$ or $\left.\mathrm{sp}^{3}\right)$ peaks are absent, implying the formation of perfluorographane (CF). The remaining peak area, about $10 \%$ of the total peak area, corresponds well to the position of C($\left.F_{2} /-F_{3}\right)$ peaks. Such double or triple functionalized carbon atoms could be present at the defect sites. In contrast to the fluorination process of FG, the good adhesion of monolayer graphene to the substrate will render diffusion of fluorine beneath the graphene sheet less probable, which results in a single-sided fluorination of $\mathrm{SG}^{7,24,25}$. From the XPS of fluorinated $\mathrm{SG}$, the major signals come from the $\mathrm{C}\left(\mathrm{sp}^{3}\right)$ and $\mathrm{C}(-\mathrm{F})$ bonds, whereas the $\mathrm{C}\left(\mathrm{sp}^{2}\right)$ bond signal (located at 284.4 $\mathrm{eV}$ ) disappears in the $\mathrm{C} 1 \mathrm{~s}$ spectrum. This indicates the complete transformation of in-plane $\mathrm{sp}^{2}$ carbon to $\mathrm{sp}^{3}$ carbon and $\mathrm{C}(-\mathrm{F})$ bonds. It is also noticed that there are two types of C(-F) bonds (locating at $\sim 288 \mathrm{eV}$ and $\sim 290 \mathrm{eV}$, respectively) observed in the XPS spectrum, both of which have been observed in previous experiments ${ }^{16}$. Similar to free-standing perfluorographane, for the SG we also observe $\sim 10 \% \mathrm{C}\left(-\mathrm{F}_{2}\right.$ and $\left.-\mathrm{F}_{3}\right)$, which can be attributed to the defect sites as well. When comparing the atomic proportion of the $\mathrm{C}(-\mathrm{F})$ and $\mathrm{C}\left(\mathrm{sp}^{3}\right)$ carbon, the ratio of one is obtained in the $\mathrm{C}(-\mathrm{F}) / \mathrm{C}\left(\mathrm{sp}^{3}\right)$ of the SG sample, indicating the formation of $\mathrm{C}_{2} \mathrm{~F}$. This result is significantly different from the conventional single-sided fluorination where an F concentration 
corresponding to $\mathrm{C}_{4} \mathrm{~F}$ is reached on supported graphene by using long time exposure of pure $\mathrm{XeF}_{2}$. This implies that by using this electron beam activated fluorination technique, a high $\mathrm{F}$ concentration can be obtained that goes beyond the conventional limit of fluorination. The comparison of the Raman spectra of the three samples is shown in Fig. 2B. The characteristic Raman signal of pristine graphene has disappeared in the case of saturated FG, which matches well with the reported perfluorographane. Raman spectrum relates mainly to the complete optical transparency of the material ${ }^{13}$. As for the SG, prominent $\mathrm{D}\left(\right.$ at $\left.\sim 1350 \mathrm{~cm}^{-1}\right)$ and $\mathrm{G}$ peaks (at $\sim 1580 \mathrm{~cm}^{-1}$ ) were observed in the Raman spectrum, whereas the 2D peak (at $\sim 2700 \mathrm{~cm}^{-1}$ ) was found to be negligible. This result is consistent with the disappearance of the XPS C( $\left.\mathrm{sp}^{2}\right)$ bond signals analysis in the SG sample.

This promising result of EBAF based local fluorination of the graphene raises a question about the obtained $\mathrm{C}_{\mathrm{x}} \mathrm{F}$ structure, its electronic structure as well as the lateral resolution that the EBAF process can achieve. To further investigate the formation of the fluorographene as well as the bandgap opening effect, additional experiments were conducted on the SG samples by varying the fluorination time. Fig. 3A exhibits the plot of fluorine concentration as a function of fluorination time (more deconvoluted XPS spectra can be found from Fig. S1-S4). From the plot, it can be seen that for shorter times, the fluorine concentration increases with the process time and the saturation occurs at 5 minutes with an atomic $F$ concentration of $\sim 35 \%$ corresponding to $\mathrm{C}_{2} \mathrm{~F}$. Due to the sufficient energy and small probe size, the electron beam has been used to remove functionalities on graphene and achieved great success in the patterning of graphene with nanometer resolutions ${ }^{10,26}$. Since in our process, the primary and secondary electrons can both dissociate the formed C-F bonds ${ }^{10}$, an electron beam activated defluorination process will be triggered when the gas flux is stopped and only apply the electron irradiation (schematic illustration can be found in Fig. S5). Here, the electron beam used in the de-fluorination process is the same as that in the fluorination process, i.e. a vacuum condition 
of $10^{-6} \mathrm{mbar}$, an electron accelerating voltage of $5 \mathrm{kV}$ and beam current of $650 \mathrm{pA}$. The detailed relation between the atomic $\mathrm{F}$ concentration and de-fluorination time is shown in Fig. 3B with the starting material being saturated $\mathrm{SG}\left(\mathrm{C}_{2} \mathrm{~F}\right)$ with the $\mathrm{F}$ concentration of $\sim 35 \%$. Here, it can be seen that the de-fluorination process is less efficient and it takes $\sim 240$ minutes to totally recovery the sample. Therefore, the combination of the electron beam activated fluorination and de-fluorination techniques constitutes a reversible approach to precisely control the fluorine concentration from pristine graphene to $\mathrm{C}_{2} \mathrm{~F}$ in the $\mathrm{SG}$ samples and back to the pristine graphene sample.

To investigate the influence of covalently bonded $\mathrm{F}$ atoms on the electronic structure of graphene, XPS valence band spectra were recorded on fluorinated SG samples with various F concentrations. The XPS valence band spectrum reflects the projected density of states (PDOS) below the Fermi level (defined as the binding energy of $0 \mathrm{eV}$ of the measured material). Thus, XPS provides an efficient tool to understand the modifications in the electronic structure of graphene with covalently bonded F atoms. Fig. 4 shows the comparison of the valence band spectra of the fluorographene. In pristine graphene, the fitted line of the valence band spectrum in the low binding energy region intersects with the energy axis at the Fermi level and this agrees well with a vanishing band gap of graphene. Another observed feature of the pristine graphene is the prominent peak at the binding energy of $\sim 8.5 \mathrm{eV}$. This energy corresponds well to the peak position of the delocalized $\pi$ orbital electrons of graphene. In contrast to pristine graphene, for the fluorinated graphene, when fitting the slope of the low energy tail of the PDOS, the line intersects the energy axis at positive energy, which indicates a band opening. The PDOS intensity close to the Fermi level vanishes. The values of the valence band maximum (VBM) obtained from the experimental curves increases with the sequence of pristine graphene, $11 \%$, $22 \%, 28 \%$ and $35 \%$ fluorographene as $0 \mathrm{eV}, 0.9 \mathrm{eV}, 1.4 \mathrm{eV}, 1.8 \mathrm{eV}$ and $2.9 \mathrm{eV}$, respectively. As for the $\pi$ state peak, the intensity gradually decreases with the atomic F concentration which 
can be related to the increased electron localization of the C-F bonds. The $\pi$ state peak is thus expected to decrease in intensity with higher F concentration as observed. For the saturated SG $\left(\mathrm{C}_{2} \mathrm{~F}\right)$ sample, the $\pi$ peak disappears. This result coincides with the disappearance of the $\mathrm{sp}^{2}$ bond component in the C1s peak observed in the XPS spectra. Theoretically, in the single-sided fluorinated graphene, the interaction between the $\mathrm{p}$ orbitals of $\mathrm{F}$ and the $\pi$ orbitals of $\mathrm{C}$ producing $\mathrm{sp}^{3}$ bonds will make the Fermi level not appear in the exact center of the bandgap. The related maximum energy shift of the Fermi level is $\sim 0.2 \mathrm{eV}^{7,27}$. Therefore, it is possible to estimate the band gap of the fluorinated SG samples from the experimental VBMs. Thus, it can be deduced that the $\mathrm{C}_{2} \mathrm{~F}$ two-dimensional (2D) material has a band gap of $5.8 \pm 0.2 \mathrm{eV}$, equal to diamond and that should be sufficiently insulating where insulating areas of graphene are needed in graphene-based devices. The $\mathrm{C}_{4} \mathrm{~F}$ exhibits a smaller bandgap of $2.8 \pm 0.2 \mathrm{eV}$ corresponding to a semiconducting 2D material. To better understand the relation between structure and electronic structure, ab initio density functional theory (DFT) calculations were performed on the single-sided fluorinated graphene (see the supporting information Fig. S7S11). From these simulations, it is found that band gap of graphene increases as the percentage of fluorine coverage increases, which is consistent with our experimental observations. Moreover, we also observe that the band gap of the fluorinated graphene $\left(\mathrm{C}_{\mathrm{x}} \mathrm{F}\right.$ where $\mathrm{x}$ is 2,4 and 8) opens only when the atomic sites of the $\mathrm{F}$ atoms are positioned as boat configurations, while the chair configurations cannot open a band gap and this is consistent with the report report $^{28}$. Together with the observed bandgap opening, this implies that the $\mathrm{F}$ atoms in the saturated single side fluorographene in this work have the potential $\mathrm{C}_{2} \mathrm{~F}$ boat structures.

A milestone in graphene research is the discovery of triangular moiré patterns in the graphene/hBN heterostructure superlattice, in which the electronic properties of graphene can be tailored through periodic moiré potentials and variety of significant phenomenon have been observed, e.g. the emergence of van Hove singularities ${ }^{29}$, Hofstadter's butterfly ${ }^{30,31}$, high- 
temperature quantum oscillations ${ }^{32}$, etc. Graphene derivatives, e.g. graphane $(\mathrm{CH})$ and fluorographene $\left(\mathrm{CF}, \mathrm{C}_{2} \mathrm{~F}\right.$ chair and $\mathrm{C}_{2} \mathrm{~F}$ boat), due to their lattice constant being slightly different from the one of graphene, can also form moiré superlattices when being well aligned and on top of monolayer graphene (the simulated moiré patterns can be found in Fig. S12). However, since after synthesis, the obtained graphene derivatives always show a rippled surface $^{25}$, the transferred graphene derivative layer cannot atomically lock to the flat graphene layer to form the moiré superlattice. To address this issue, our strategy is to fluorinate the topmost layer in the exfoliated bilayer graphene directly and to achieve the $\mathrm{C}_{2} \mathrm{~F} /$ graphene superlattice structure. First of all, to understand the stacking order, scanning tunneling microscopy imaging (Fig. 5A) was carried out on the highly oriented pyrolytic graphite (HOPG) from which the bilayer graphene is exfoliated, and the triangular lattice indicates the typical Bernal stacking order of the graphite. The EBAF technique was applied on the exfoliated bilayer graphene which was then imaged by using the AFM. It is noted in the AFM experiment that the patterns start to appear after a long time ( 8 hours) scanning in a comparably large scale as shown in Fig. 5B. When we performed fine scanning at a higher magnification as shown in Fig. $5 \mathrm{C}$, the moiré pattern consisting of bright and dark rectangular patterns is clearly visible and the denoted unite cell shows distances of $13.8 \mathrm{~nm}$ and $6.8 \mathrm{~nm}$ in two directions perpendicular to each other. This generated moiré patterns agree very well with our simulated moiré patterns arising from $\mathrm{C}_{2} \mathrm{~F}$ boat/graphene structure (see supporting information Fig. S12a). They are clearly distinct from the triangular moiré patterns obtained in hBN/graphene system and also would be expected, following our moiré simulations (Fig. S12b), in $\mathrm{C}_{2} \mathrm{~F}$ chair/graphene and $\mathrm{CF} /$ graphene bilayer. To the best of our knowledge, this is the first experimental observation of the rectangular moiré patterns arising from the $\mathrm{C}_{2} \mathrm{~F}$ boat/graphene superlattice (the detailed structure can be found in Fig. S13), which means that a new type of periodic electrostatic moiré potential has been generated. This materials system has the potential to reveal novel interesting properties of $2 \mathrm{D}$ materials since the interaction between the two $2 \mathrm{D}$ 
materials layers will be very distinct from the hitherto dominating graphene/BN moiré materials. The fluorine can be attached to the graphene either in the boat or in the chair configuration. We have simulated the structure of the one-sided saturated $\mathrm{C}_{2} \mathrm{~F}$ for both, the chair and the boat configuration (see supporting information Table S1) and found that for the boat configuration, the total energy per fluorine atom is $1.5 \mathrm{eV}$ lower than that for the chair configuration. Thus, the boat configuration is energetically more stable, which is in good agreement with our experimental analysis.

Owing to the intrinsic property of the focused electron beam, the here demonstrated electron beam activated synthesis method is also a controllable, local and free writing technique, and thus provides the possibility to fabricate lateral semiconducting/insulating nanopatterns directly on the conductive graphene surface. To demonstrate this, we further employed the technique to fabricate sharp $\mathrm{C}_{2} \mathrm{~F}$ and $\mathrm{C}_{4} \mathrm{~F}$ nanostrips directly on a graphene and measured the relative conductivity of the different layers by conductive AFM (Fig. 6A). It can be seen from the contrast in the image that the insulating $\mathrm{C}_{2} \mathrm{~F}$ nanostrips has the lowest conductivity with the current close to zero nA and the graphene shows the highest conductivity, while the $\mathrm{C}_{4} \mathrm{~F}$ nanostrips have an intermediate conductivity. To further demonstrate the resolution of this technique, we have used the AFM adhesion mapping. This technique is based on the fact that the adhesion forces between the AFM tip and graphene will change after fluorination, thus providing the contrast in the maps between graphene and the $\mathrm{C}_{2} \mathrm{~F}$ layer. Fig. $6 \mathrm{~B}$ shows a $15 \mathrm{~nm}$ $\mathrm{C}_{2} \mathrm{~F}$ line directly fabricated on monolayer graphene, which can best indicate the resolution of the technique. It should also be mentioned that the interfacial width in Fig. 6A (90\% to $10 \%$ criterion) is $9 \mathrm{~nm}$.

\section{I I . CONCLUSION}

In conclusion, electron beam activated fluorination is shown to be a highly efficient technique to obtain a local fluorination and de-fluorination of graphene. The structuring of $\mathrm{C}_{\mathrm{x}} \mathrm{F}$ 
nanostructures is possible with high resolution of $9-15 \mathrm{~nm}$ both with respect to chemical composition as well as spatial resolution. The saturated fluorination on the FG leads to a stoichiometric fluorographene of $\mathrm{CF}$, while on the $\mathrm{SG}$ sample to an insulating $\mathrm{C}_{2} \mathrm{~F}$ was approached which has overcome the conventional limit of semiconducting $\mathrm{C}_{4} \mathrm{~F}$. The concentration of the covalently bonded fluorine can be precisely controlled and results in a quantitative band gap engineering of graphene. Moreover, this local nano-synthesis method constitutes a novel 2D lithography technique to freely write semiconducting and insulating nanopatterns directly on conductive graphene surface with $15 \mathrm{~nm}$ resolution. These findings pave the way to fabricate graphene-based flexible and transparent electronic nanodevices.

\section{SUPPLEMENTARY MATERIAL}

See the supplementary material for details on the XPS spectra, DFT models, moiré pattern simulations, process parameters and field-effect transistor performances.

\section{ACKNOWLEDGEMENT}

We acknowledge the Swedish Research Council (Vetenskapsrådet) (621-2012-3679, 201605259), Knut and Alice Wallenberg Foundation (KAW Graphene) (Swedish Graphene Initiative) and Formas Foundation (2019-01538) for the financial support. We are also grateful to Swedish National Infrastructure for Computing (SNIC) and the PRACE-2IP project CHARTERED2 resource Salomon cluster based in Czech Republic at the IT4Innovations for computer support.

\section{APPENDIX: EXPERIMENTAL}

Graphene samples: Large scale monolayer graphene samples were synthesized by chemical vapor deposition technique and transferred to $\mathrm{Cu}$ TEM grid and $\mathrm{SiO}_{2}(285 \mathrm{~nm}) / \mathrm{Si}$ substrate. Prior to the experiments, the samples were immersed in chloroform for $24 \mathrm{~h}$ and then blow dried under $\mathrm{N}_{2}$ to remove the contaminations. Finally, the cleaned samples were checked by light optical microscope, XPS and Raman. Bilayer graphene are exfoliated from highly oriented 
pyrolytic graphite and transferred onto $\mathrm{SiO}_{2}$ substrate and characterized through optical microscope and Raman spectroscopy.

Graphene fluorination: The local electron beam activated graphene fluorination and defluorination were conducted in a FEI Strata DB235 FIB/SEM at a vacuum condition of $10^{-6}$ mbar under the electron acceleration voltage of $5 \mathrm{kV}$ and beam current of $650 \mathrm{pA}$ (more details on the electron dose can be found in Fig. S14). The $\mathrm{XeF}_{2}$ molecules were injected through an equipped gas injection system (GIS) inside FIB/SEM chamber with a partial pressure at the exit of the gas nozzle of $600 \mathrm{~Pa}^{33}$. The fluorination was carried out under the electron beam irradiation with simultaneous $\mathrm{XeF}_{2}$ molecule injection, while the de-fluorination was performed only under electron beam irradiation.

Characterizations: A Physical Electronics Quantum 2000 Scanning ESCA microprobe was used to conduct the X-ray photoelectron spectroscopy (XPS) experiments with a $\mathrm{Al} \mathrm{K \alpha} \mathrm{X}$-ray (1486.7 eV) source. In the XPS measurements, due to the low conductivity of the fluorographene, a shift of the XPS spectra move to the higher energy region took place, thus an electron gun neutralizer was open to compensate this charging effect. The Raman measurements were performed by using a Renishaw inVia Raman microscope with a $532 \mathrm{~nm}$ wavelength laser. The AFM was carried out in a Bruker Multimode 8 AFM under the peakforce quantitative nanomechanical mapping mode and conductive AFM mode ${ }^{9,34-38}$.

DFT calculation: Ab initio density functional theory (DFT) calculations are performed by using a plane wave based pseudo potential method (VASP) to study the single sided fluorination of monolayer graphene ${ }^{39,40}$. We have used the generalized gradient method of Perdew, Burke and Ernzerhof for the exchange-correlation functional along with PAW potential ${ }^{41,42}$. The structures were optimized using conjugate gradient method with forces calculated from Hellman-Feynman theorem. The energy and force thresholds are kept at $10^{-5} \mathrm{eV}$ and $10^{-2} \mathrm{eV} / \AA$, respectively. For geometry optimization, a $5 \times 5 \times 1$ Gamma centered $\mathbf{k}$ grid was used. For total energies and electronic structures, we have used a $15 \times 15 \times 1$ Gamma centered $\mathbf{k}$ grid. 
The electronic structure of the $\mathrm{C}_{n} \mathrm{~F}$ structures $(n=2,4,8,16,32)$ were investigated. These structures represent respectively $50 \%, 25 \%, 12.5 \%, 6.25 \%$ and $3.125 \%$ single sided fluorination coverage of monolayer graphene (see supporting information Fig. S7-S11).

\section{REFERENCES}

${ }^{1}$ S.M. Hollen and J.A. Gupta, Science. 352, 415 (2016).

${ }^{2}$ L. Wang, C.R. Dean, I. Meric, K.L. Shepard, J. Hone, P. Kim, K. Watanabe, and T. Taniguchi, Science. 342, 1 (2013).

${ }^{3}$ S. Park and R.S. Ruoff, Nat. Nanotechnol. 4, 217 (2009).

${ }^{4}$ N. Wang, M.K. Samani, H. Li, L. Dong, Z. Zhang, P. Su, S. Chen, J. Chen, S. Huang, G. Yuan, X. Xu, B. Li, K. Leifer, L. Ye, and J. Liu, Small 14, 1801346 (2018).

${ }^{5}$ J. Mindemark, S. Tang, H. Li, and L. Edman, Adv. Funct. Mater. 28, 1801295 (2018).

${ }^{6}$ K.S. Novoselov, Science. 306, 666 (2004).

${ }^{7}$ J.T. Robinson, J.S. Burgess, C.E. Junkermeier, S.C. Badescu, T.L. Reinecke, F.K. Perkins, M.K. Zalalutdniov, J.W. Baldwin, J.C. Culbertson, P.E. Sheehan, and E.S. Snow, Nano Lett. 10, $3001(2010)$.

${ }^{8}$ R. Stine, W.-K. Lee, K.E. Whitener, J.T. Robinson, and P.E. Sheehan, Nano Lett. 13, 4311 (2013).

${ }^{9}$ H. Li, L. Daukiya, S. Haldar, A. Lindblad, B. Sanyal, O. Eriksson, D. Aubel, S. HajjarGarreau, L. Simon, and K. Leifer, Sci. Rep. 6, 19719 (2016).

${ }^{10}$ F. Withers, T.H. Bointon, M. Dubois, S. Russo, and M.F. Craciun, Nano Lett. 11, 3912 (2011).

${ }^{11}$ M. Dubois, K. Guérin, Y. Ahmad, N. Batisse, M. Mar, L. Frezet, W. Hourani, J.-L. Bubendorff, J. Parmentier, S. Hajjar-Garreau, and L. Simon, Carbon. 77, 688 (2014).

${ }^{12}$ H. Zhang, L. Fan, H. Dong, P. Zhang, K. Nie, J. Zhong, Y. Li, J. Guo, and X. Sun, ACS Appl. Mater. Interfaces 8, 8652 (2016).

${ }^{13}$ R.R. Nair, W. Ren, R. Jalil, I. Riaz, V.G. Kravets, L. Britnell, P. Blake, F. Schedin, A.S. 
Mayorov, S. Yuan, M.I. Katsnelson, H.-M. Cheng, W. Strupinski, L.G. Bulusheva, A. V

Okotrub, I. V Grigorieva, A.N. Grigorenko, K.S. Novoselov, and A.K. Geim, Small 6, 2877 (2010).

14 J.E. Johns and M.C. Hersam, Acc. Chem. Res. 46, 77 (2013).

${ }^{15}$ H.Y. Liu, Z.F. Hou, C.H. Hu, Y. Yang, and Z.Z. Zhu, J. Phys. Chem. C 116, 18193 (2012).

${ }^{16}$ W. Feng, P. Long, Y.Y. Feng, and Y. Li, Adv. Sci. 3, 1 (2016).

${ }^{17}$ F. Karlický and M. Otyepka, J. Chem. Theory Comput. 9, 4155 (2013).

${ }^{18}$ Y. Liang and L. Yang, MRS Proc. 1370, mrss11 (2011).

${ }^{19}$ X. Li, W. Cai, J. An, S. Kim, J. Nah, D. Yang, R. Piner, A. Velamakanni, I. Jung, E. Tutuc, S.K. Banerjee, L. Colombo, and R.S. Ruoff, Science 324, 1312 (2009).

${ }^{20}$ X. Zhang, L. Wang, J.H. Xin, B.I. Yakobson, and F. Ding, J. Am. Chem. Soc. 136, 3040 (2014).

${ }^{21}$ X. Li, Y. Zhu, W. Cai, M. Borysiak, B. Han, D. Chen, R.D. Piner, L. Colomba, and R.S. Ruoff, Nano Lett. 9, 4359 (2009).

${ }^{22}$ I.H. Krouse and P.G. Wenthold, Inorg. Chem. 42, 4293 (2003).

${ }^{23}$ M. Liao and Q. Zhang, J. Phys. Chem. A 10647 (1998).

${ }^{24}$ S. Kumar, D. Parks, and K. Kamrin, ACS Nano 10, 6552 (2016).

${ }^{25}$ D.C. Elias, R.R. Nair, T.M.G. Mohiuddin, S. V. Morozov, P. Blake, M.P. Halsall, A.C. Ferrari, D.W. Boukhvalov, M.I. Katsnelson, A.K. Geim, and K.S. Novoselov, Science. 323, 610 (2009).

${ }^{26}$ W.K. Lee, K.E. Whitener, J.T. Robinson, and P.E. Sheehan, Adv. Mater. 27, 1774 (2015).

${ }^{27}$ H. Åžahin, M. Topsakal, and S. Ciraci, Phys. Rev. B - Condens. Matter Mater. Phys. 83, 1 (2011).

${ }^{28}$ H. Şahin, M. Topsakal, and S. Ciraci, Phys. Rev. B 83, 115432 (2011).

${ }^{29}$ D.I. Indolese, R. Delagrange, P. Makk, J.R. Wallbank, K. Wanatabe, T. Taniguchi, and C. Schönenberger, Phys. Rev. Lett. 121, 137701 (2018). 
${ }^{30}$ B. Hunt, J.D. Sanchez-Yamagishi, A.F. Young, M. Yankowitz, B.J. LeRoy, K. Watanabe, T. Taniguchi, P. Moon, M. Koshino, P. Jarillo-Herrero, and R.C. Ashoori, Science. 340, 1427 (2013).

${ }^{31}$ C.R. Dean, L. Wang, P. Maher, C. Forsythe, F. Ghahari, Y. Gao, J. Katoch, M. Ishigami, P. Moon, M. Koshino, T. Taniguchi, K. Watanabe, K.L. Shepard, J. Hone, and P. Kim, Nature 497, 598 (2013).

${ }^{32}$ R.K. Kumar, X. Chen, G.H. Auton, A. Mishchenko, D.A. Bandurin, S. V. Morozov, Y. Cao, E. Khestanova, M. Ben Shalom, A. V. Kretinin, K.S. Novoselov, L. Eaves, I. V. Grigorieva, L.A. Ponomarenko, V.I. Fal'Ko, A.K. Geim, R. Krishna Kumar, X. Chen, G.H. Auton, A. Mishchenko, D.A. Bandurin, S. V. Morozov, Y. Cao, E. Khestanova, M. Ben Shalom, A. V. Kretinin, K.S. Novoselov, L. Eaves, I. V. Grigorieva, L.A. Ponomarenko, V.I. Fal'Ko, and A.K. Geim, Science. 357, 181 (2017).

${ }^{33}$ M. Tramšek and B. Žemva, Acta Chim.Slov 53, 105 (2006).

${ }^{34}$ F. Sun, H. Li, K. Leifer, and E.K. Gamstedt, Int. J. Solids Struct. 129, 40 (2017).

${ }^{35}$ A. Lundstedt, R. Papadakis, H. Li, Y. Han, K. Jorner, J. Bergman, K. Leifer, H. Grennberg, and H. Ottosson, Small Methods 1, 1700214 (2017).

${ }^{36}$ H. Li, R. Papadakis, S.H.M. Jafri, T. Thersleff, J. Michler, H. Ottosson, and K. Leifer, Commun. Phys. 1, 44 (2018).

${ }^{37}$ J. Liu, Y. Wang, H. Li, S. Costil, and R. Bolot, J. Mater. Process. Technol. 249, 471 (2017).

38 J. Liu, R. Papadakis, and H. Li, Appl. Phys. Lett. 113, 083108 (2018).

${ }^{39}$ G. Kresse and J. Furthmüller, Phys. Rev. B 54, 11169 (1996).

${ }^{40}$ T. Physik, T.U. Wien, and W. Hauptstrasse, Phys. Rev. B 47, 558 (1993).

${ }^{41}$ J. Perdew, K. Burke, and M. Ernzerhof, Phys. Rev. Lett. 77, 3865 (1996).

${ }^{42}$ P.E. Blöchl, Phys. Rev. B 50, 17953-17979 (1994). 


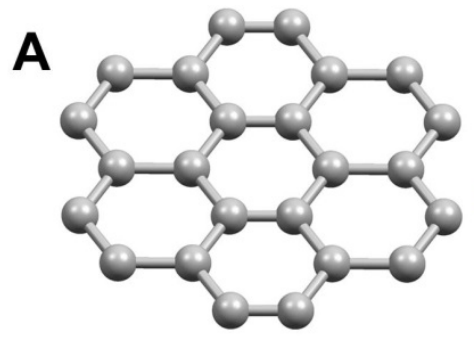

B

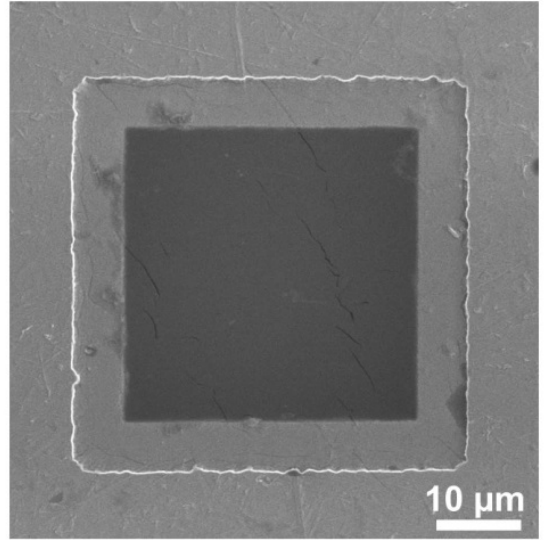

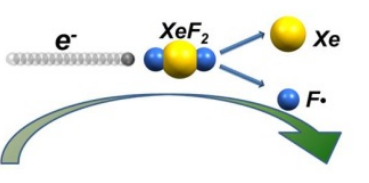

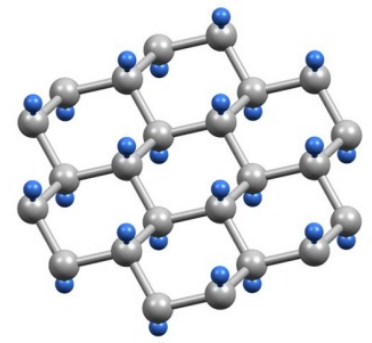

C

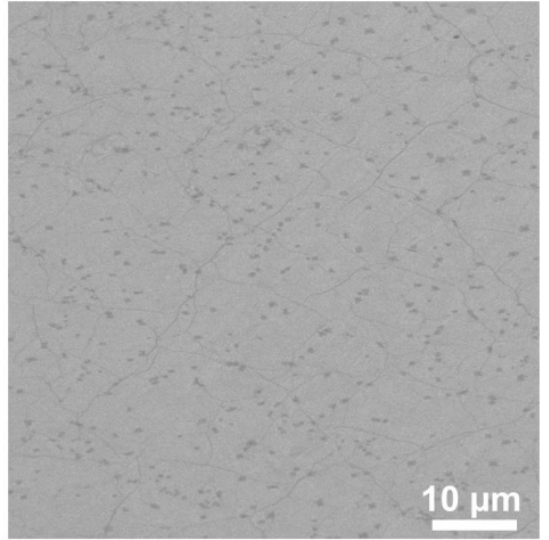

FIG 1. Schematic and graphene samples. (A). Illustration of the electron beam activated fluorination technique. SEM images of the free standing graphene on TEM grid (B) and supported graphene on $\mathrm{SiO}_{2} / \mathrm{Si}$ substrate (C).
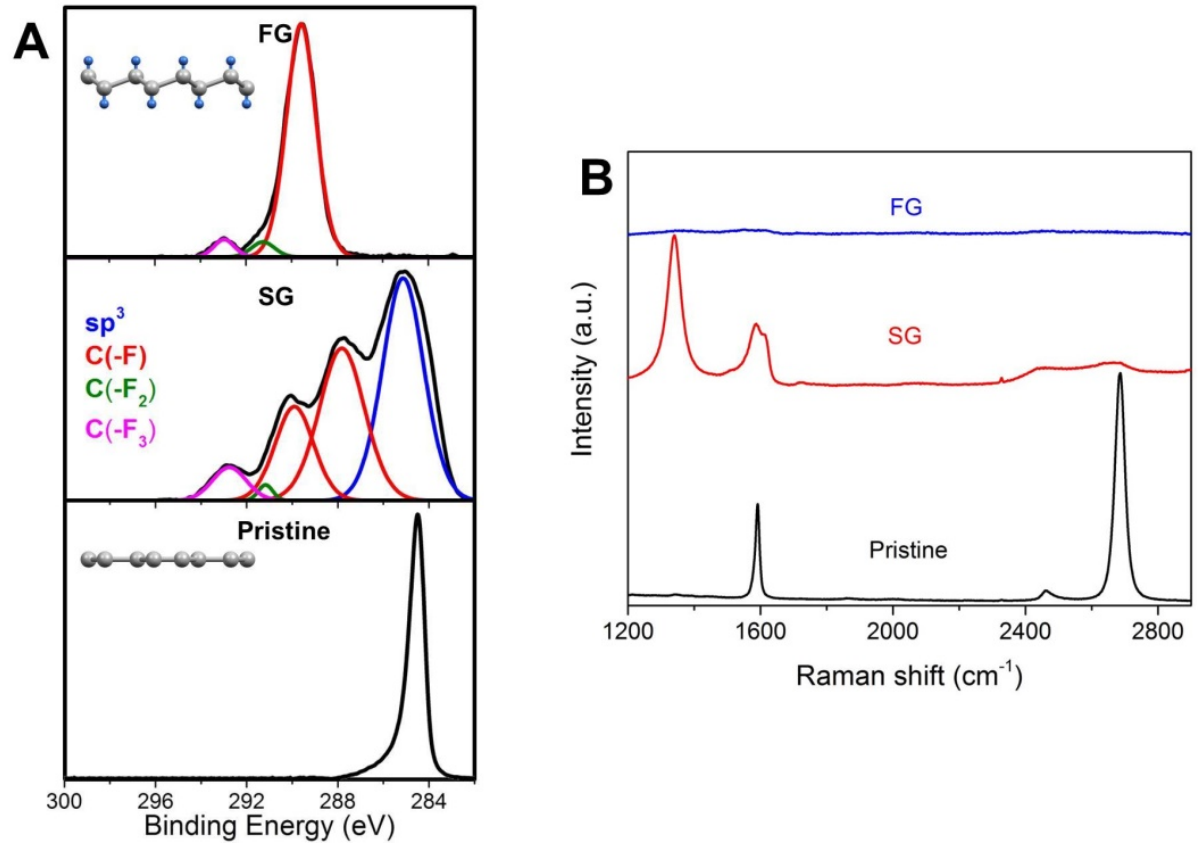

FIG 2. Spectroscopic characterization of fluorographene. XPS C 1s (A) and Raman (B) comparisons of the perfluorographane $(\mathrm{CF})$, single-side fluorinated graphene $\left(\mathrm{C}_{2} \mathrm{~F}\right)$ and pristine 
graphene. The inserted graph in (A) is the atomic side-view structure of the perfluorographane (CF) and pristine graphene analyzed from XPS.
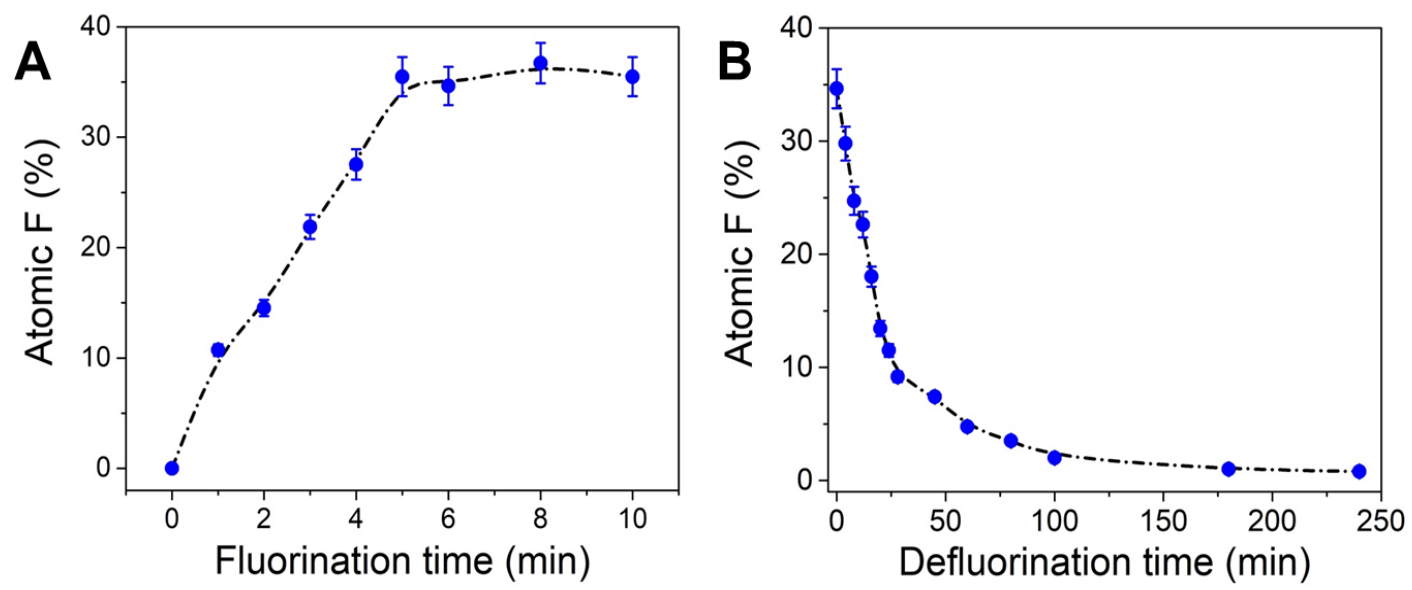

FIG 3. Fluorine concentration as a function of applied electron dose with standard deviation as error bar and fitted line. (A) Electron beam induced fluorination and (B) electron beam induced defluorination.

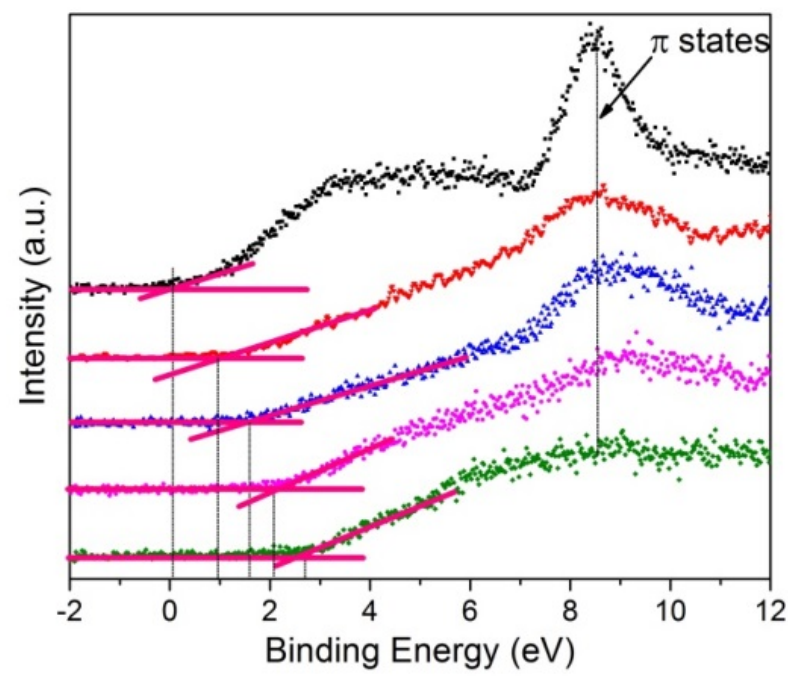

FIG 4. Valence band spectra of single-side fluorographene. The Fermi level is located at the binding energy of $0 \mathrm{eV}$. From top to bottom, the F concentrations are $0,12 \%, 22 \%, 28 \%$ and $35 \%$, respectively. 

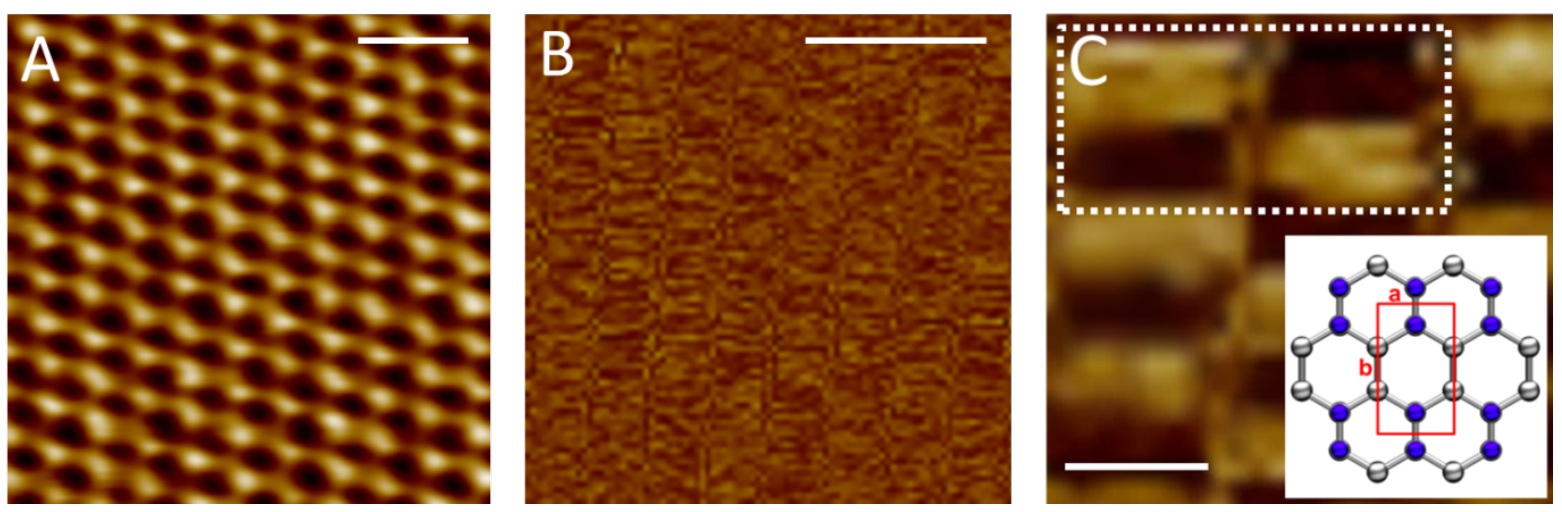

FIG 5. Moiré pattern characterizations. (A) Scanning tunneling microscopy image of the HOPG sample for the double layer graphene exfoliation, where the triangular lattice indicates the Bernal stacking in the HOPG. The scale bar is $5 \AA$. (B) large scale atomic force microscopy (AFM) image of the single side fluorinated bilayer graphene, where block patterns appears. The scale bar is $20 \mathrm{~nm}$. (C) Fine scanning of the atomic force microscopy image of the single side fluorinated bilayer graphene and the dot rectangle denotes the unit cell with the length and width of $13.8 \mathrm{~nm}$ and $6.8 \mathrm{~nm}$, respectively. The right inserted is the plan view of the structure of the $\mathrm{C}_{2} \mathrm{~F}$ boat with double lattice pentameters. The lengths of indications of $\mathrm{a}$ and $\mathrm{b}$ are $2.49 \AA \AA$ and $4.36 \AA$, respectively, which equals to a mismatch of $1.2 \%$ and $2.3 \%$, respectively, compared to pristine graphene. The scale bar is $5 \mathrm{~nm}$. 


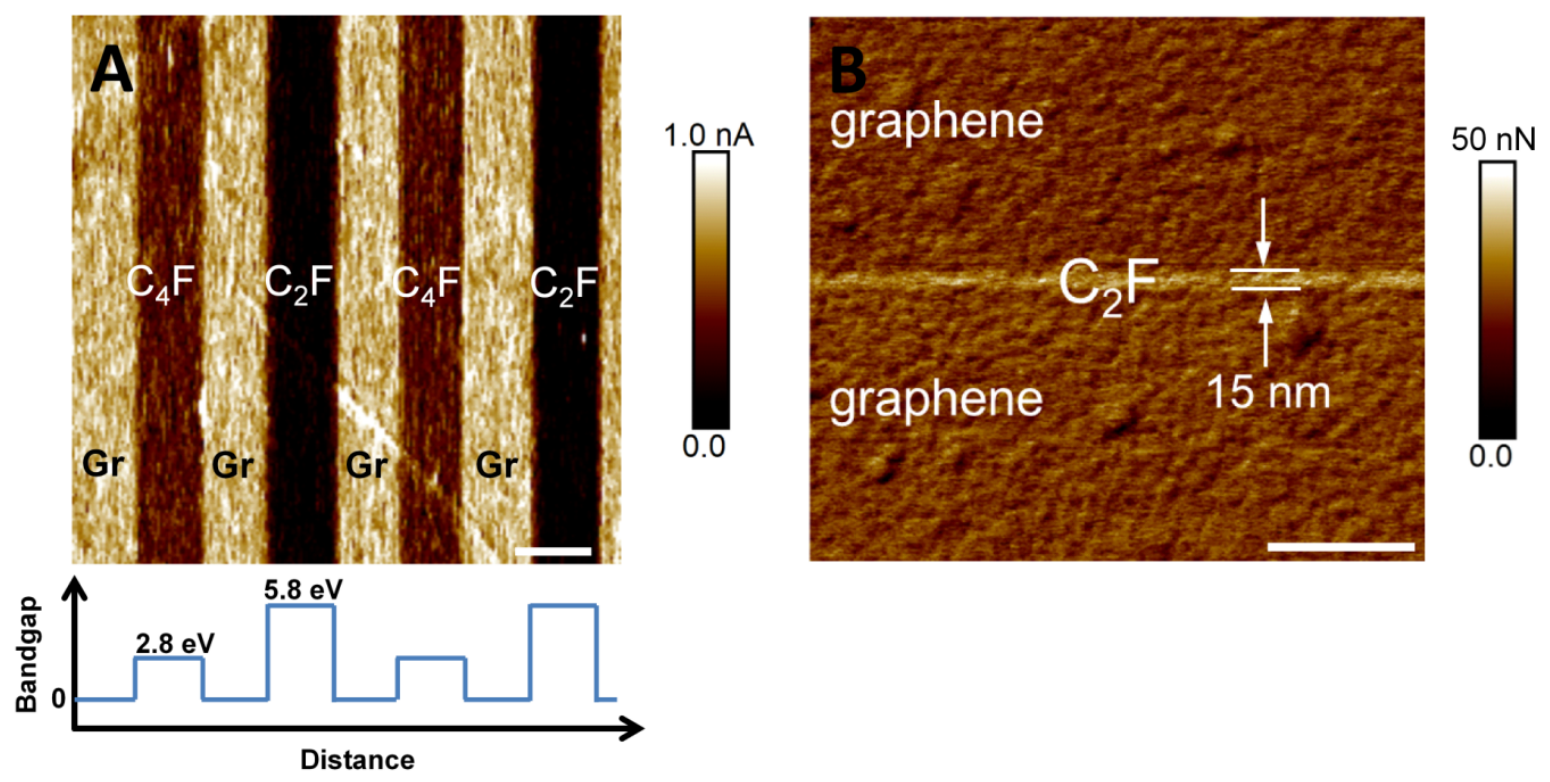

FIG 6. AFM mappings of the fluorinated nanopatterns. (A) AFM conductive mapping nanopatterns consisting of graphene, semiconducting $\mathrm{C}_{4} \mathrm{~F}$ and insulating $\left(\mathrm{C}_{2} \mathrm{~F}\right)$ stripes directly fabricated on monolayer graphene. The contrast of the mapping indicates the decreasing of the conductivity with the increased fluorine concentration. The scale bar is $100 \mathrm{~nm}$. (B) AFM adhesion mapping of the $\mathrm{C}_{2} \mathrm{~F}$ line on graphene surface, which indicates the resolution of the technique is measured as $15 \mathrm{~nm}$. The scale bar is $100 \mathrm{~nm}$. 